\title{
La maqueta como herramienta de aprendizaje para desarrollo de maquinaria agrícola.
}

\section{The mock up as a learning tool for developing agricultural machinery}

\author{
Rafael R. Sola-Guirado*, Jesus A. Gil-Ribes, Juan Agüera-Vega, Sergio Castro- \\ García, Emilio González-Sánchez; Gregorio L. Blanco-Roldan
}

Fecha de recepción: 03/06/2019; Fecha de revisión: 15/07/2019; Fecha de aceptación: 03/09/2010

Cómo citar este artículo:

Sola-Guirado, R.R., Gil-Ribes, J.A., Agüera-Vega, J., Castro-García, S., González-Sánchez, E. \& BlancoRoldán, G.L. (2019). La maqueta como herramienta de aprendizaje para desarrollo de maquinaria agrícola. Revista de Innovación y Buenas Prácticas Docentes, 8(3), 64-73.

*Autor de Correspondencia: ir2sogur@uco.es

\begin{abstract}
Resumen:
Se propone la introducción de un ejercicio basado en proyectos en la asignatura de "Diseño de maquinaria agraria. Automática agraria". Para resolver el problema los alumnos han de pasar por todas las fases de las metodologías de desarrollo de productos: búsqueda de información, planificación del trabajo, diseño, cálculo, fabricación, etc. Al final, los alumnos deberán obtener una maqueta que modele el trabajo de una máquina agrícola con funciones cargadora o manipuladora. Los alumnos eligieron una metodología de trabajo en grupo por ingeniería secuencial, en lugar de la aconsejada por ingeniería concurrente, dado que requiere menor interacción entre ellos, pero al final del ejercicio expusieron los problemas encontrados habiendo aprendido de los pros y contras de ambas. Los indicadores finales de la práctica muestran la mejora de la calidad docente con mayor participación del alumnado y con mejores resultados académicos. Sin embargo, se requiere del profesorado una dedicación y tiempo extra en la asignatura, así como mayores recursos, por lo que en el futuro se debería intentar la coordinación de la práctica con otras materias similares o con cursos de formación permanente.
\end{abstract}

Palabras clave: maqueta; proyectos; máquina; diseño;

\begin{abstract}
:
An exercise based on project is proposed in lesson "Agricultural machinery design. Automation". To solve the problem, students must go through all phases of product development methodologies: information search, work planning, design, calculation, manufacturing... Finally, they must obtain a model which simulates the work performed by a agricultural machinery with loader or manipulator functions. This year, the students chose a methodology of work sequentially, instead of the one recommended, concurrent engineering. This choice required less interaction among them but at the end they exposed the cons and pros of using it. The teaching indicator show improvement of the quality of the teaching with greater participation of students, and better academic results. However, teachers are required an extra dedication and time, as well as more resources. So, in the future should be coordinated the practice with other similar subjects or extra courses.
\end{abstract}

Key Words: mock-up; project; machine; design; 


\section{INTRODUCCIÓN}

Una de las herramientas más clarificadoras para la asimilación de los conocimientos adquiridos por los alumnos de ingeniería, es la aplicación de ejercicios reales que les permitan afrontar las adversidades que se le plantean, generar hipótesis para su resolución y tomar decisiones. La metodología del "trabajo por proyectos" permite que el alumno se integre en una realidad más compleja y global (Chandrasekaran et al., 2013), a la vez que logra adquirir conocimientos en el proceso de resolución del problema, abarcando gran parte del contenido de la asignatura (Jakubiak, 2016). Este modo de trabajo es una valiosa herramienta para que el alumno pueda percibir el aprendizaje de un modo más atractivo e interactúe con colegas de su entorno (Lehmann et al., 2008). En este proceso, además, se requiere poner en práctica otras habilidades adquiridas con otras asignaturas a lo largo de la titulación (García \& Álvarez, 2015). Esta interdisciplinaridad posibilita planificar actividades con una perspectiva unificada para su aplicación práctica (de Graaff \& Kolmos, 2007). Numerosas experiencias en universidades abalan los aspectos positivos de esta técnica de enseñanza que se puede abordar con distintas tipologías (Chandrasekaran et al. 2012).

Para llevar a cabo los proyectos, es necesario establecer unos requisitos mínimos y plantear un breve guión, pero el grado de seguimiento en mayor o menor medida no ha de ser fijo, pues depende de la capacidad del alumno (Willey, 1983). Las técnicas de Proceso Orientado al Aprendizaje de Consultas Guiadas fomentan un ambiente de trabajo y cooperación en pequeños grupos de 3 o 4 estudiantes que focalizan sus actividades, cuidadosamente preparadas, para profundizar en conceptos y su comprensión (Moog, 2014). Sin embargo, un proceso de guía muy estricto limita sus competencias de aprendizaje, restando importancia al fundamento de autoaprendizaje, fundamental en el trabajo por proyectos (Loyens, 2008). Los nuevos marcos educativos proponen compartir la responsabilidad del profesor, dando el control a los propios estudiantes ante escenarios que les obligan a una mayor implicación, convirtiéndolos en sujetos activos de construcción y gestión de su conocimiento (LagoaVarela et al., 2016). Para ello, el estudiante aprenderá del profesor y de sus propios compañeros formando equipos activos y cooperacionales. Esta tendencia ayudará a aportar al alumnado las competencias exigidas en su marco laboral.

Otro aspecto relevante de la aplicación práctica de problemas de ingeniería es la necesidad de utilización de herramientas informáticas que faciliten su resolución de manera más eficiente. En los últimos años, se está produciendo una importante revolución de software en que el conocimiento generado por diversidad de disciplinas teóricas se está plasmando en herramientas que permiten dar solución a una infinidad de problemas aplicados e ingenieriles (Maibaum, 2000). El modo en que el profesorado puede resolver problemas de manera más eficiente y eficaz condiciona que el tipo de clases que imparte sea diferente a la enseñanza tradicional. Como tal, es esencial que el alumnado las conozca y pueda manejar software específico que le permitan desarrollar ciertas habilidades para el posterior desempeño profesional. En la actualidad, se están llevando a cabo estudios que exponen la mejora de ciertas habilidades y la adquisición de otras nuevas, en un entorno de aprendizaje basado en software, en ingenierías (Seffah \& Grogono, 2002). 
Con estas premisas, en el presente proyecto se plantea al alumnado la resolución de un trabajo que aborda los contenidos curriculares de la asignatura "Diseño de maquinaria agraria. Automática agraria" ( $4^{\circ}$ curso, Obligatoria, Itinerario de Ingeniería Rural, Grado de Ingeniería Agroalimentaria y del Medio Rural). Hay un problema concreto para la enseñanza de estas materias como es la falta de créditos para desarrollarlas, ya que, nunca han sido propiamente vinculadas al sector agrario. Pero la evolución actual del sector y, sobre todo, de la maquinaria agrícola, cada vez dotada de más elementos oleohidráulicos, electrónicos y de control, ha determinado la necesidad de abordar estas temáticas y el mercado laboral exige técnicos con esta formación. Por tanto, dada la necesidad y la limitación temporal, la mejor forma de hacerlo es mediante la combinación teórico-práctica, pero no en el sentido tradicional de prácticas dirigidas, sino mediante el trabajo en base a Proyectos, que permitan la autonomía del alumno y su organización junto con otros compañeros. Se utilizará un enfoque constructivista en el tratamiento interdisciplinar de las actividades planteadas, en el que los alumnos deberán pasar por las diferentes fases de diseño y desarrollo de productos (DDP), que acabará en la construcción de un prototipo de máquina agrícola (Ayağ, 2016).

\section{DESARROLLO DE LA EXPERIENCIA DE INNOVACIÓN}

\subsection{Diseño de la metodología de trabajo}

Se planteó un proyecto para la asignatura que consistió en la planificación, diseño, fabricación y automatización de un prototipo (maqueta) que simule una máquina con funciones de manipulación (Figura 1) (cargador frontal, manipuladora telescópica, etc.) y que debe de realizar una tarea determinada. Estas máquinas son muy empleadas en el sector agrícola, disponiendo de una conjunción de sistemas mecánicos, oleohidráulicos y eléctricos, lo que justifica su elección como ejemplo para el proceso de enseñanza-aprendizaje de esta materia. El prototipo por desarrollar debía trasladar un objeto de peso y dimensiones determinados (paquete de pañuelos) desde un punto inicial en el suelo hasta otro situado a $10 \mathrm{~cm}$ a su derecha y $10 \mathrm{~cm}$ de altura y no podrá superar las dimensiones de 40×40×40 cm ni el peso de $2 \mathrm{~kg}$.

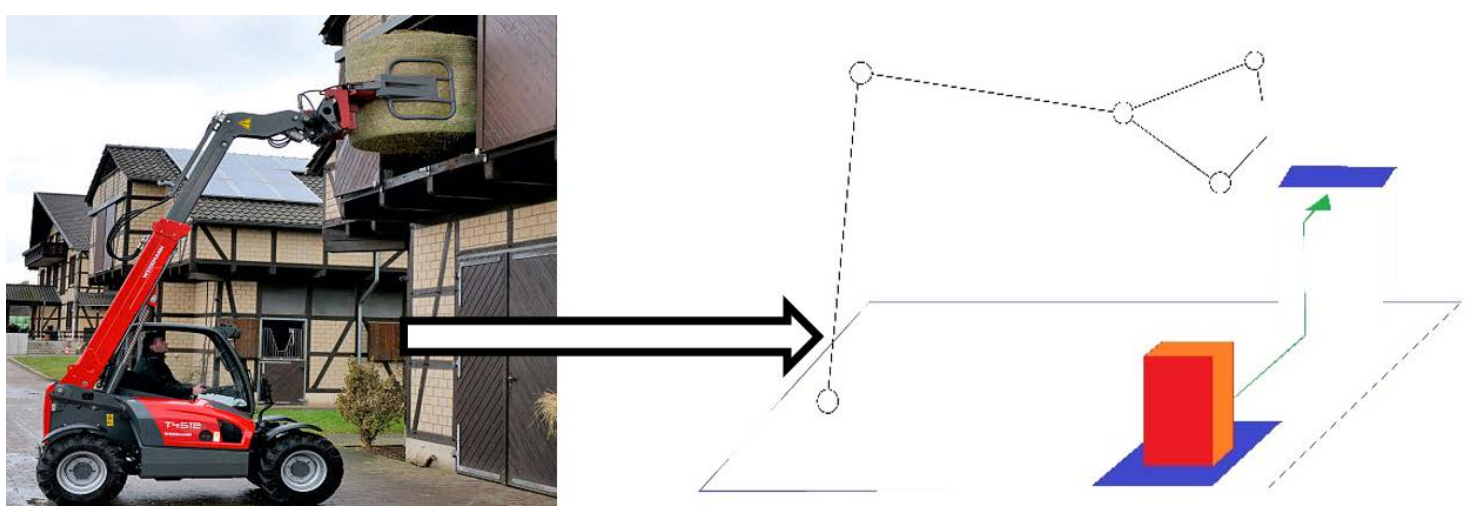

Figura 1. Máquina cargadora telescópica y esquema del problema planteado.

Para resolver la práctica propuesta los alumnos formaron un grupo, asemejando una empresa, y repartieron las tareas necesarias para la resolución del problema pudiendo elegir cualquiera de las metodologías de Diseño explicadas en clase: Ingeniería Secuencial o Ingeniería Concurrente (Figura 2). En el primer caso, las tareas van pasando de alumno a alumno de manera independiente condicionando las decisiones tomadas del anterior al resto. En el segundo caso, los alumnos tienen una iteración constante y pueden tomar decisiones comunes en cada una de las fases intervinientes en el proceso de diseño. En cualquier caso, deberán de pasar por todas las fases que implica un DDP explicada en la asignatura: Estudio de la técnica, planificación del trabajo, diseño conceptual y de detalle, cálculo, documentación de 
taller, taller, ensamblaje y puesta en marcha. A modo de ejemplo de la metodología de DDP, los alumnos realizarán una visita a empresas de fabricación de maquinaria agrícola para ver en primera persona los modos de trabajo. Al final de la práctica, los alumnos expusieron una presentación con los resultados obtenidos en las diferentes fases que fue evaluada por los profesores.

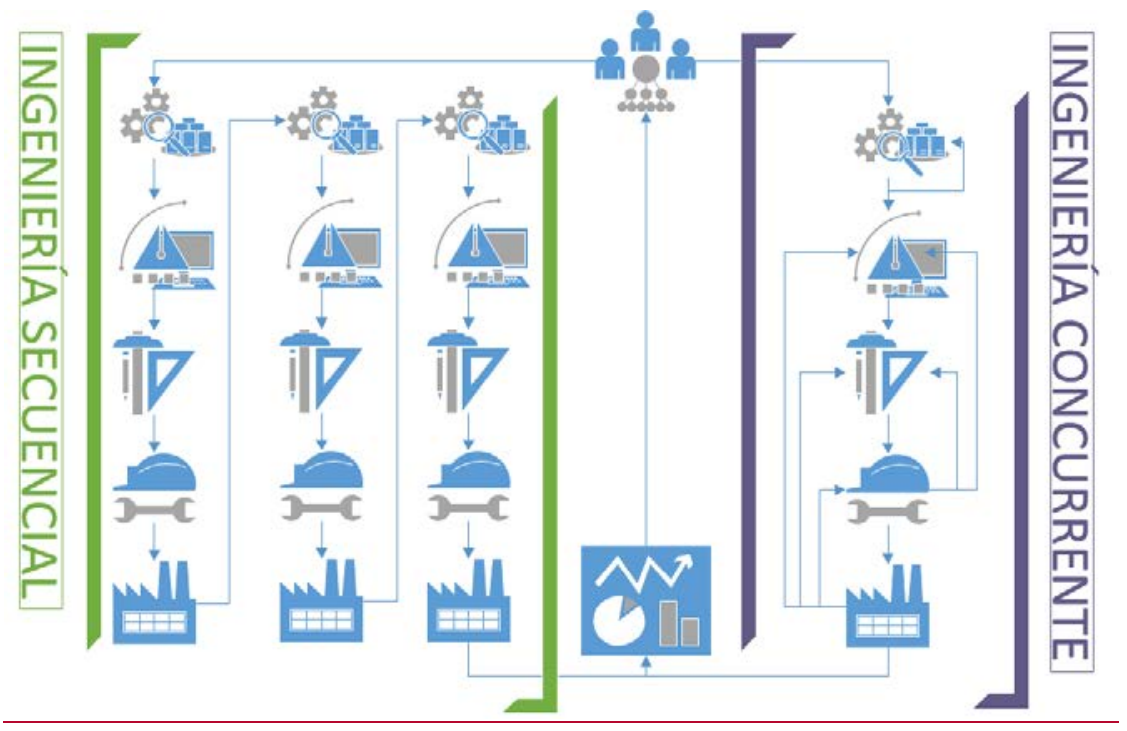

Figura 2. Metodologías de diseño y grado de relación de las actividades intervinientes.

Fuente: Elaboración propia

\subsection{Objetivos}

El objetivo general es conseguir que el alumnado aplique los conocimientos adquiridos en la asignatura "Diseño de maquinaria agraria. Automática agraria", mediante la resolución de un problema práctico planteado como un Proyecto y en un proceso de colaboración con varios compañeros, adquiriendo las competencias expuestas a continuación. Además, se pretende mejorar la calidad de la asignatura en su ámbito docente.

\subsection{Competencias desarrolladas}

Las competencias que abarca esta propuesta metodológica se exponen en la Figura 3.

\begin{tabular}{ll}
\hline Comptencia a adquirir & Causas para adquirirla \\
\hline $\begin{array}{l}\text { CB2 Capacidad de resolución de problemas creatividad, iniciativa, metodología y } \\
\text { razonamiento crítico. }\end{array}$ & $\begin{array}{l}\text { Para generar una solución eficiente, deberá } \\
\text { enfrentarse un problema y.razonar un } \\
\text { procedimiento. }\end{array}$ \\
\hline $\begin{array}{l}\text { CB4 Capacidad para la búsqueda y } \\
\text { utilización de la normativa y reglamentación } \\
\text { relativa a su ámbito de actuación }\end{array}$ & $\begin{array}{l}\text { Para buscar la mejor alternativa a su } \\
\text { planteamiento, deberá investigar al respecto. }\end{array}$ \\
\hline $\begin{array}{l}\text { CU2 Conocer y perfeccionar el nivel de } \\
\text { usuario en el ámbito de las TIC. }\end{array}$ & $\begin{array}{l}\text { Para conseguir un producto final, el } \\
\text { estudiante debe usar diferentes tipos de } \\
\text { tecnologías y software. }\end{array}$ \\
\hline
\end{tabular}

Figura 3. Competencias necesarias para abordar la práctica docente y causas para adquirirla. Fuente: Elaboración propia. 


\begin{tabular}{|c|c|}
\hline Comptencia a adquirir & Causas para adquirirla \\
\hline $\begin{array}{l}\text { CEC7 Capacidad para conocer, comprender } \\
\text { y utilizar los principios de ingeniería del } \\
\text { medio rural: cálculo de estructuras, y } \\
\text { construcción, hidráulica, motores y } \\
\text { máquinas, electrotecnia, proyectos técnicos. }\end{array}$ & $\begin{array}{l}\text { Para poder dar una solución es necesrio } \\
\text { conocer la teoría de estrucutras, máquinas } \\
\text { así como plantear un proyecto determinado. }\end{array}$ \\
\hline $\begin{array}{l}\text { CEC9 Capacidad para conocer, comprender } \\
\text { y utilizar los principios de: Toma de } \\
\text { decisiones mediante el uso de los recursos } \\
\text { disponibles para el trabajo en grupos } \\
\text { multidisciplinares }\end{array}$ & $\begin{array}{l}\text { Para llevar a cabo todas las fases del } \\
\text { proyecto, deberán tomar desiones y } \\
\text { colaborar íntimamente entre compañeros. }\end{array}$ \\
\hline $\begin{array}{l}\text { CEMC4 Capacidad para conocer, } \\
\text { comprender y utilizar los principios de: } \\
\text { Mecanización agraria. Motores y máquinas } \\
\text { agrícolas. Características y diseño de } \\
\text { maquinaria para instalaciones agrarias. } \\
\text { Automática agraria. }\end{array}$ & $\begin{array}{l}\text { Para que el producto cumpla los requisitos } \\
\text { planteados es necesario poner en práctica } \\
\text { los principios desarrollados en el campo del } \\
\text { disñeo de maquinaria y automática. }\end{array}$ \\
\hline
\end{tabular}

\subsection{Evaluación de la experiencia}

Los alumnos realizaron una matriz DAFO para obtener las principales debilidades, amenazas, fortalezas y oportunidades que haya ofrecido esta práctica. De la exposición realizada en clase, el profesorado evaluó el grado de adquisición de las competencias adquiridas con la experiencia, y dichas fueron comparada con aquellas adquiridas por otros dos grupos de estudiantes en dos cursos académicos anteriores que utilizaron un planteamiento de enseñanza tradicional basado en clases magistrales. Para evaluar la incidencia del proyecto en el programa docente y la asignatura, los 4 docentes que la imparten realizarán una evaluación de los principales indicadores sobre la mejora de la calidad en la docencia, indicando un valor numérico: mucho (4), bastante (3), poco (2) o nada (1).

\section{RESULTADOS Y DISCUSIÓN}

Los alumnos eligieron trabajar según la metodología de ingeniería secuencial, esto es, se reparten las tareas entre ellos según las fases a entregar y realizan varias iteraciones, pero sin interactuar entre ambos. La elección de este modelo, según su decisión, vino motivada por sus preferencias de trabajar de manera individual el lugar de crear sesiones de trabajo colaborativo. Esta tendencia confirma las dificultades que aún representa entre los estudiantes la realización de trabajar en grupos, dificultando la adquisición de la competencia de colaboración.

Los resultados que han extraído los alumnos de las diferentes fases se muestran a continuación:

- FASE 1. Se realizó una búsqueda de información de máquinas similares a las planteadas en el problema como investigación previa. A pesar, de todas las bases de datos disponibles en la universidad, la principal herramienta de búsqueda fue ${ }^{\circledR G o o g l e . ~}$ En base a ello, se produjo una lluvia de ideas de los mecanismos, materiales y actuadores más convenientes para el problema. De las mismas se extrajo la posibilidad de realizar un mecanismo de pantógrafo para mantener la horizontalidad de una pinza de apertura en los movimientos de subida y bajada. En cuanto a los actuadores de los movimientos, se eligió la posibilidad de utilizar hidráulica en lugar de mecanismos eléctricos, por lo que se requería principalmente cilindros. Se encontraron maquetas 
similares en el que usaban jeringuillas para simular este sistema. Como material más ligero y fácil de manejar en la fabricación, se eligió la madera.

- FASE 2. Con los resultados del equipo anterior, otro equipo realizó un presupuesto de los medios y materiales finales necesarios para su consecución. Finalmente, diseñaron un cronograma o diagrama Gant con el software ${ }^{\circledR}$ Microsoft Project para planificar el trabajo con una estructura secuencial o de salto de vaya, esto es, cada equipo hacía una actividad y pasaba los resultados al siguiente equipo sin interactuar y consensuar decisiones.

- FASE 3. Con las premisas de los equipos anteriores, se crearon unos bocetos del mecanismo que pasaron a la siguiente fase. Una vez pasaron por la segunda iteración, se definió el diseño de detalle con un modelo CAD (Figura 4) de la solución, con todos las partes de la máquina mediante un software de modelado (®SolidWork 2010, Dassault Systèmes).

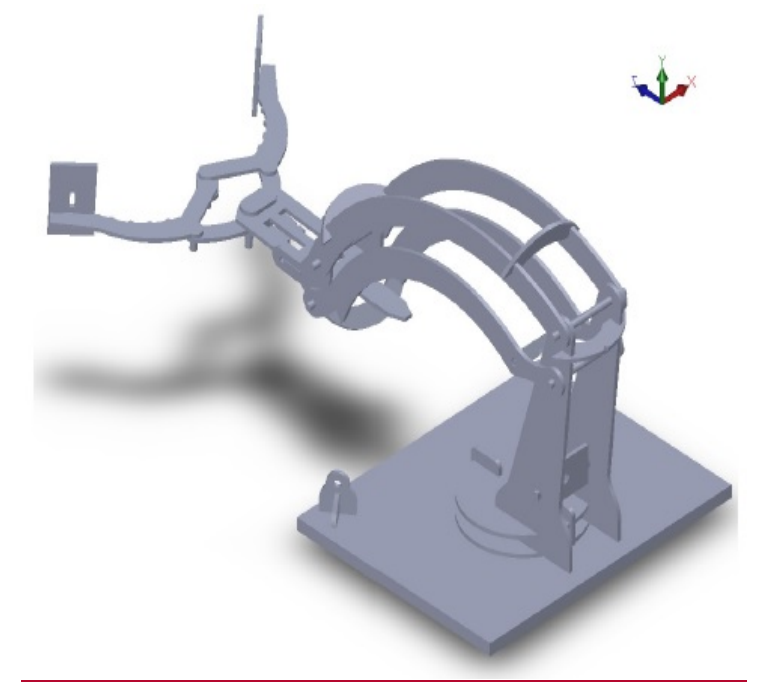

Figura 4. Modelo CAD de una de las máquinas diseñadas por los alumnos. Fuente: Captura del programa ${ }^{\circledR S}$ Solidwork

- $\quad$ FASE 4. Con los bocetos del mecanismo implicado, se realizaron una serie de cálculos de los puntos conflictivos en el problema (tensiones, recorridos, diseño hidráulico, etc.). En esta fase, algunos alumnos utilizaron un módulo específico de cálculo del sofware ${ }^{\circledR S o l i d W o r k . ~ C o n ~ l o s ~ r e s u l t a d o s ~ m i ́ n i m o s ~ s e ~ d i m e n s i o n a r o n ~ a l g u n o s ~}$ de los elementos para poder trasladarlos de nuevo a otra iteración (Figura 5). 


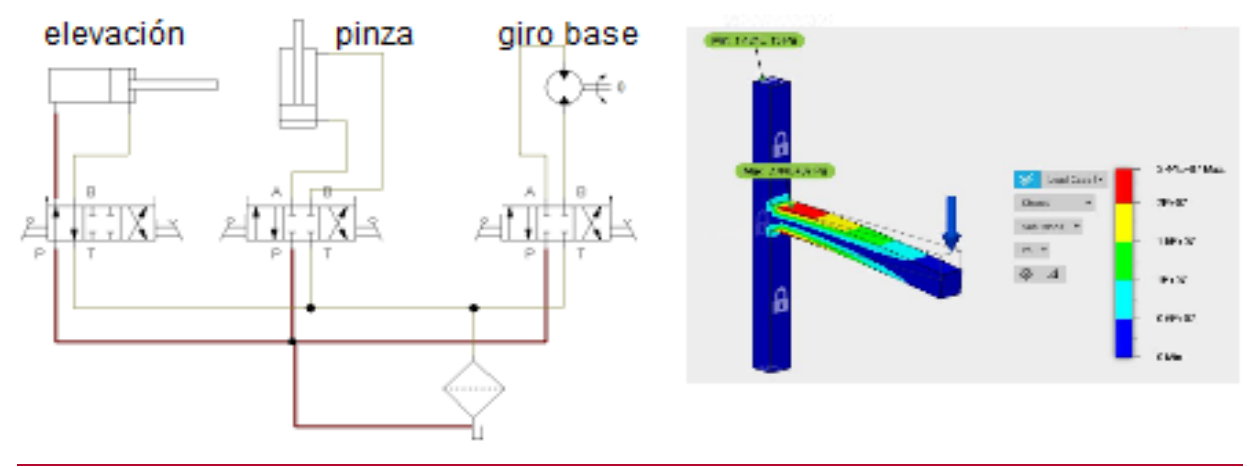

Figura 5. Ejemplos de esquemas y cálculo realizados por los alumnos en su problema. Esquema hidráulico del mecanismo y cálculo estructural de brazo de la pinza en voladizo. Fuente: Cuaderno de trabajo de los alumnos.

- $\quad$ FASE 5. Una vez congelado el diseño, se generó la documentación de taller, es decir, unos planos técnicos de los componentes a montar, una de lista de materiales para su abastecimiento y una serie de órdenes de trabajo y planificación de procesos a realizar. Algunas de las piezas fueron obtenidas mediante una fresadora $2 \mathrm{D}$ donde el código CNC fue obtenido de los planos del modelo anterior mediante el software ${ }^{\circledR V}$ Vetric.

- FASE 6. En esta fase se debía haber realizado una automatización del ejercicio, con elementos de control. Sin embargo, sólo se realizó una propuesta práctica en la que los movimientos se realizarían con servomotores controlados por una placa de Arduino, donde se programarían los movimientos con el software ${ }^{\circledR}$ Arduino IDE.

- $\quad$ FASE 7. Finalmente, todos los componentes fueron ensamblados y puestos a punto, realizándose una demostración en clase del funcionamiento del prototipo (Figura $6)$.

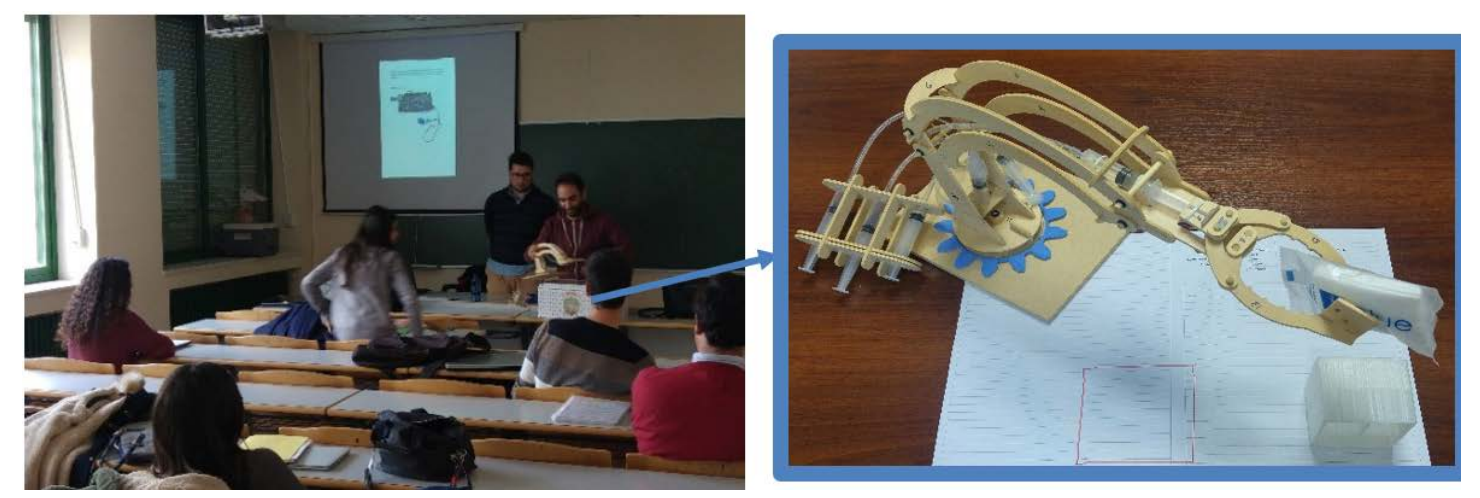

Figura 6. Ensamblaje y puesta a punto del prototipo diseñado. Fuente: Imágenes tomadas en el aula donde se imparte clase (UCO. 
El resultado de la matriz DAFO de los alumnos se muestra en la Figura 7:

\section{DEBILIDADES}

- El acabado de los componentes no es óptimo.

- El trabajo secuencial limita a las decisiones que se tomen en la fase anterior.

- No se recuerdan bien algunos conceptos cursados en otras asignaturas (física, hidráulica, resistencia de materiales, etc.)

\section{AMENAZAS}

- Subestimar el proyecto por tratarse de una maqueta.

- Desconocer si los grupos de las fases posteriores mantendrán tu idea.

- Tiempo limitado en la asignatura y necesidad de trabajo en casa.

- Hay que aprender las diferentes herramientas de software implicadas en las fases.

\section{OPORTUNIDADES}

- Comprender de manera práctica los mecanismos.

- Poner en marcha un proyecto real.

- Comprender de primera mano las posilidades que hubiera supuesto el trabajo en ingeniería concurrente.

- Utilizar y aprender sofware específico que no se cursa en ninguna materia de la carrera.

Figura 7. Recopilación de indicadores de valor en la práctica docente, según una matriz DAFO realizada por los alumnos, tras su curso.

Fuente: Elaboración propia.

Los resultados de mejora en los indicadores de mejora docente, con la introducción de este proyecto, se muestran en la Figura 8.

\begin{tabular}{lcc}
\hline & Puntuación media (del 1 (nada) al 4 (mucho) \\
\hline ¿Con la práctica planteada, ha mejorado .... & profesorado & alumnado \\
\hline$\ldots$ el uso de las tutorías? & 2 & 3 \\
\hline ... asistencia a clase? & 2 & 2 \\
\hline ... el interés por la compresión de las explicaciones.? & 2 & 2 \\
\hline ... la comunicación alumno-profesor? & 2 & 3 \\
\hline ... el clima de trabajo y participación del alumnado? & 3 & 3 \\
\hline ... el cumplimiento de los objetivos de la asignatura? & 3 & - \\
\hline ... la coordinación de las actividades entre profesorado? & 3 & - \\
\hline ... las notas finales con respecto a otros años? & 2 & - \\
\hline \multicolumn{4}{c}{ Figura 8. Cuestionario realizado a los alumnos y profesores tras la práctica docente y } \\
\multicolumn{4}{c}{ puntuación media obtenida. } \\
Fuente: Elaboración propia.
\end{tabular}


En general, se percibe que ha mejorado levemente el interés del alumnado, su participación y asistencia, así como la interacción con el profesorado. Además, la obligación de a hacer un trabajo grupal, ha mejorado la comunicación entre los alumnos, aunque finalmente eligieran el modo trabajo de menor cooperación. Todo ello, se ha plasmado en una mejora del rendimiento de los alumnos en sus notas finales. La evaluación del profesorado de las competencias adquiridas por los estudiantes manifiesta resultados más favorables que respecto a los cursos anteriores con la enseñanza mediante lecciones magistrales. Este hecho recalca la importancia de la experimentación sobre problemas reales en la mejora del aprendizaje del alumno.

A nivel docente, se ha mejorado la coordinación de actividades y objetivos entre el profesorado del área. La necesidad de adaptar levemente la estructura de la asignatura y dotarla con un enfoque más práctico, ha conllevado la necesidad de una mayor interacción. Sin embargo, la implementación de prácticas similares requiere un esfuerzo extra dentro y fuera del aula y el empleo de mayor tiempo. Además, la disposición de recursos materiales y software por parte de la universidad ha sido fundamental para que el alumno pueda realizar la práctica sin dificultades añadidas.

\section{CONCLUSIONES}

La introducción del ejercicio ha resultado de gran utilidad para desarrollar y aplicar la mayor parte de los contenidos teóricos de la asignatura utilizada. El enfoque del problema como un proyecto global permite cubrir de manera práctica los contenidos de la asignatura, dinamizando su curso y haciendo más ameno el trabajo de los alumnos en casa. La experiencia ha permitido cubrir un gran número de competencias sin forzar la preparación de otras actividades que permitan adquirirlas de manera más aislada. Las competencias generales expuestas han mejorado con respecto a años anteriores. Los alumnos han utilizados nuevos software y recursos que reconocen no hubieran manejado, si no hubieran cursado en esta práctica académica.

Ante la elección del modo de trabajo en equipo, los alumnos eligen el modo que exige menor cooperación, pero al final del trabajo, los alumnos reconocieron las limitaciones que ello ha supuesto frente a un modo de trabajo basado en ingeniería concurrente. Afrontar el proyecto de esta manera ha permitido que el alumno comprenda las carencias derivadas de este modo de trabajo, reconociendo de manera global que un proceso más interactivo habría mejorado sus resultados con un poco más de esfuerzo extra. Esto ha llevado, a que la resolución técnica de la práctica no haya sido excelente y a que algunas fases como la automatización, no se haya realizado por falta de coordinación.

Se ha producido una mejora en los indicadores docentes de comunicación con el alumnado, y participación dentro y fuera de clase. La coordinación horizontal entre los profesores del área ha mejorado, aunque se ha invertido mayor número de recursos y tiempo por parte de los docentes. Las limitaciones de tiempo y recursos que requiere el problema pueden ser un hándicap para llevar a cabo su resolución con un grado de éxito mayor, aunque los alumnos admiten que es una oportunidad para utilizar nuevos medios que no podrían aprender con la enseñanza tradicional. En este sentido, una potencial mejora podría pasar por integrar la práctica con asignaturas afines a la misma o complementar los contenidos y software de la práctica con cursos de formación permanente.

\section{REFERENCIAS}

Ayağ, Z. (2016). An integrated approach to concept evaluation in a new product development. Journal of Intelligent Manufacturing, 27(5), 991-1005. 
Chandrasekaran, S., Stojcevski, A., Littlefair, G. \& Joordens, M. (2012) "Learning through projects in engineering education". En Proceedings of the 40th SEFI Annual Conference, Brussels.

Chandrasekaran, S., et al. (2013). Project-oriented design-based learning: aligning students' views with industry needs. International Journal of Engineering Education, 29(5),1109-1118.

de Graaff, E. \& Kolmos, A. (2007). "History of problem-based and project-based learning! En Management of change. Brill Sense, 1-8.

García, A. T., \& Álvarez, A. P. (2015) Cooperación científica en la integración de las TIC al proceso de formación de ingenieros y arquitectos. Revista Referencia Pedagógica, 3(1), 82.

Jakubiak, O. (2016). The pros and cons of Problem-Based Learning compared to the traditional foreign language teaching methods. Pedagogika, 25(1), 313-322.

Lagoa-Varela, M., Álvarez García, B., \& Boedo Vilabella, L. (2016). The Bologna process: a study about teachers' perception of their new role and its consequences. En 2nd. International conference on higher education advances (HEAD'16) (pp. 486-494). Valencia: Editorial Universitat Politècnica de Valencia.

Lehmann, M., Christensen, P., Du, X., \& Thrane, M. (2008). Problem-oriented and project-based learning (POPBL) as an innovative learning strategy for sustainable development in engineering education. European Journal of engineering education, 33(3), 283-295.

Loyens, S., Magda, J., Rikers, R. (2008). Self-directed learning in problem-based learning and its relationships with self-regulated learning. Educational Psychology Review, 20(4), 411-427.

Moog, R. (2014). Process oriented guided inquiry learning. En M. R. McDaniel, R. Frey, S. Fitzpatrick, \& H.L. Roediger (Eds), Integrating cognitive science with innovative teaching in STEM disciplines (pp. 148-150). Washington: Washington University Libraries.

Seffah, A., \& Grogono, P. (2002). Learner-centered software engineering education: From resources to skills and pedagogical patterns. En Proceedings 15th Conference on Software Engineering Education and Training. IEEE, 14-21.

Wiley, K. (1983). Effects of a self-directed learning project and preference for structure on self-directed learning readiness. Nursing Research, 32(3), 181-185. 